\title{
Fragmentación y cooperación en la gobernanza energética global*
}

\author{
GONZALO ESCRIBANO \\ Universidad Nacional de Educación a Distancia (UNED), Facultad de CC.EE., c/ Senda del Rey, 11, \\ 28040 Madrid, España.E-mail: gescribano@cee.uned.es
}

\begin{abstract}
RESUMEN
El artículo aborda la interdependencia energética y la gobernanza mundial de la energía desde una perspectiva de economía política internacional. Describe la naturaleza fragmentada de la gobernanza energética, así como la tendencia hacia una mayor inter-polaridad, analizando por qué la hegemonía está limitada por la interacción de una miríada de regímenes internacionales energéticos dedicados a aspectos diferentes que conforman un 'complejo de regímenes energéticos' en lugar de un único régimen internacional. Por último, el trabajo analiza la gobernanza mundial de la energía desde el prisma de la provisión de bienes públicos globales, evaluando el impacto sobre la misma de las capacidades materiales, ideacionales e institucionales internacionales.
\end{abstract}

Palabras clave: Gobernanza energética, regímenes internacionales energéticos, economía política internacional.

\section{Fragmentation and Cooperation in Global Energy Governance}

\begin{abstract}
This article addresses energy interdependency and global energy governance from an international political economy perspective. It starts by describing the fragmented nature of energy governance and the trend towards increased interpolarity. It also explains why hegemony is bounded by the interplay of the myriad of dedicated international energy regimes, stating that they conform an 'energy regime complex' rather than a single international energy regime. Finally, the paper analyses the institutional evolution of the energy regime complex, trying to assess the impact of ideational, material capabilities and international energy institutions' in global energy governance.
\end{abstract}

Keywords: Energy Governance, International Energy Regimes, International Political Economy.

Códigos JEL: F53, F55, F64

\footnotetext{
* El autor agradece el apoyo recibido del Plan Nacional de I+D+i del Ministerio de Economia y Competitividad al proyecto EU-IANUS: "The EU in an unsettled international system. Crisis, multipolarity and multilateralism" (CSO2012-33361) y los comentarios recibidos en la presentación al LX Congreso de la Asociación Española para La Economía Energética (AEEE), Madrid 3 y 4 de febrero de 2014, IE Business School. El contenido del artículo es, no obstante, responsabilidad exclusive de su autor.
}

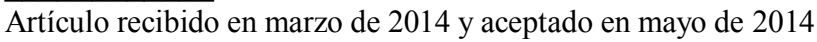

Artículo disponible en versión electrónica en la página www.revista-eea.net, ref. ə-32301 


\section{INTRODUCCIÓN}

Este artículo aborda la gobernanza energética global desde una perspectiva de economía política internacional y provisión de bienes públicos globales. Mientras que los asuntos energéticos globales han tendido a ser analizados principalmente a través del prisma geopolítico, existe una creciente literatura sobre los diferentes aspectos de la gobernanza mundial de la energía. En ella se aborda el tema tanto desde una perspectiva horizontal como temática que abarca desde la cooperación entre productores y consumidores a lucha contra el cambio climático y la pobreza energética, pasando por el buen gobierno de los recursos energéticos (Abbott, 2012; Baker y Stoker, 2013; Carbonnier, 2011; Cherp et al., 2011; Dubash y Florini, 2011; Florini y Sovacool, 2009, 2011; Goldthau y Witte, 2009, 2010 y 2011; Goldthau y Sovacool, 2012; Karlsson-Vinkhuyzen, 2010; Lesage et al., 2010a y b; Van de Graaf, 2013). Junto con la aparición de nuevos productores y consumidores mundiales, esta agenda en expansión ha hecho que la gobernanza global de la energía se convierta en un campo de estudio mucho más complejo que la simple gestión de la interdependencia entre los productores de petróleo de la OPEP y los consumidores de la OCDE (Florini y Dubash, 2011).

El artículo pretende contribuir a esa nueva literatura sobre gobernanza energética desde una perspectiva de economía política internacional que proporcione una valoración integral de tales complejidades y un marco coherente para analizar la cooperación y la discordia en los asuntos energéticos globales. En esa línea, sigue las aportaciones iniciales de autores como Kehoane (1984) y su análisis de la Agencia Internacional de la Energía ( AIE $^{1}$ ) como régimen internacional de los países industriales importadores de petróleo, sus desarrollos recientes incorporando el concepto de 'complejo de regímenes' (Colgan et al., 2013), y sus aplicaciones al caso de la UE (Escribano, 2010 y 2011; Marín Quemada et al., 2011; Mañé, 2006; Correljé y Van der Linde, 2006).

A ello añade un desarrollo de la agenda de investigación consistente en considerar la gobernanza energética global como un problema de provisión de bienes públicos globales o regionales (Escribano y García-Verdugo, 2011; Goldthau, 2012), pero no limitando el análisis a la seguridad energética sino incluyendo otros bienes públicos como la transparencia, la sostenibilidad o el desarrollo energético, cuya demanda ha aumentado considerablemente en las últimas décadas, dejando el actual sistema de gobernanza energética global obsoleto en su estructura y en su respuesta a dicha demanda. Para ello, la primera sección analiza la naturaleza fragmentada de la gobernanza mundial de la

\footnotetext{
${ }^{1}$ El Anexo 1 recoge el conjunto de arreglos institucionales, formales o informales, mencionados en el artículo, junto con una breve descripción de su objeto, naturaleza y evolución. Salvo las instituciones más conocidas, como la OPEP o la AIE, los acrónimos mantienen las siglas inglesas.
} 
energía y cómo ha evolucionado a lo largo de las últimas décadas, durante las cuales se han incluido tanto nuevos temas como nuevos actores que han aumentado la inter-polaridad energética. La segunda sección recurre al concepto de 'complejo de regímenes energéticos' para analizar su modelo de gobernanza, mientras que la tercera se centra en la provisión de bienes públicos energéticos globales. El apartado final presenta las consideraciones finales.

\section{UNA GOBERNANZA ENERGÉTICA FRAGMENTADA}

La gobernanza global se puede abordar desde diferentes enfoques de economía política internacional. Keohane (1984) sostiene que las instituciones internacionales eficaces (regímenes internacionales sólidos) tienden a surgir cuando los conflictos de intereses son más bien escasos y, sobre todo, cuando el poder o las capacidades materiales están concentrados en un grupo de actores con preferencias homogéneas. Este es ciertamente el caso del comercio internacional y los regímenes financieros, cuyos regímenes internacionales (OMC, FMI y Banco Mundial) nacen de la hegemonía estadounidense, pero resultan lo suficientemente resistentes y dependientes de la senda como para sobrevivir "después de la hegemonía" (Keohane, 1984; Rathbun, 2011). De hecho, ambos se han abordado desde la perspectiva de la provisión de bienes públicos tales como el libre comercio y la estabilidad financiera (Kaul et al., 1999; Fiorentini y Montani, 2012; Joyce y Sandler, 2008). Cox (1996) identifica tres dimensiones cuya alineación explicaría la hegemonía de un actor dado: las capacidades materiales, las ideas y las instituciones internacionales. Este marco resulta útil porque tiene que ver con el clásico problema de economía política internacional de la hegemonía.

Sin embargo, cuando el poder está fragmentado, la jerarquía es difusa y los conflictos de intereses abundan, la falta de incentivos claros para la cooperación no siempre permite el surgimiento de internacionales homogéneos (Oye, 1986; Krasner, 1983). De hecho, la energía y más específicamente el régimen de los consumidores de petróleo fue el tema sobre el cual Keohane (1984, cap. 10) construyó su argumento de qué pasaría con la cooperación internacional "después de la hegemonía" estadounidense. La creación de la OPEP en 1960 y su decisión de nacionalizar los recursos de hidrocarburos, anunciado en 1968, pusieron fin al control de las compañías petroleras de Estados Unidos sobre los recursos mundiales de crudo y de gas, que entrañaban objetivos tanto económicos como políticos (Wirl , 2012). A partir de esa fecha, la hegemonía entre los productores de crudo estuvo compartida entre productores OPEP y productores no OPEP.

Dentro de la propia OPEP, la hegemonía siempre se ha disputado entre 'palomas' y 'halcones', y no hay una evidencia terminante acerca de su capacidad para actuar como un cartel, que para mucho autores es como mucho limitada 
(Gil-Alana y Barros, 2011; Goldthau y Witte, 2011). La Organización de Países Árabes Exportadores de Petróleo (OAPC), una organización que se superpone a la OPEP, fue la que inició la crisis del petróleo de 1973, pero desde entonces los proveedores no OPEP han aumentado su participación en la producción mundial de crudo, con actores emergentes importantes como los productores de aguas profundas (Brasil, África Occidental) y no convencionales (EEUU y Canadá) que han entrado en el mercado en los últimos años.

Pese a ello, el mercado del petróleo sigue liderado por Arabia Saudí, que actúa como productor de último recurso, al ser el único productor con capacidad ociosa suficiente para estabilizar el mercado y compensar la pérdida de producción en otros lugares, ya sea debido a la guerra civil en Libia, al embargo a Irán o a las huelgas en Venezuela o Nigeria (Mann, 2012). Esta capacidad ociosa ha sido interpretada por Goldthau (2012) como un bien público suministrado por Arabia Saudita, que es quien costea su mantenimiento, aunque lo haga por intereses nacionales; el mismo autor caracteriza la seguridad de los corredores marítimos globales, desde Hormuz a Malacca, de bien público suministrado por Estados Unidos bajo el circunspecto free-riding de los consumidores asiáticos y europeos.

En cuanto al gas natural, la coordinación de los productores brilla por su ausencia. El Foro de Países Exportadores de Gas (GECF) reúne a los principales productores de gas del mundo, pero ha fracasado en su intento de lograr una cooperación significativa respecto a la producción o los precios. Esto es en parte debido a la rigidez del mercado del gas, basado en mercados regionales y contratos a largo plazo indexados a los precios del petróleo (Wagbara, 2007). Pero también al hecho de que las reservas de gas están distribuidas de manera más uniforme que las del petróleo y es por tanto menos propenso a traducirse en posiciones hegemónicas globales. Por el contrario, el mercado del gas está liderado por potencias regionales, como Rusia en Europa Central, Argelia en la Europa mediterránea o los países del Golfo Pérsico en Asia.

Por el lado de la demanda los consumidores también han tratado de cooperar para responder a los retos planteados por la OPEP. La Agencia Internacional de la Energía (AIE) fue creada para coordinar las respuestas de los países miembros de la OCDE a la crisis del petróleo de 1973. Al igual que ocurre con la OPEP, la cooperación en el seno de la AIE no siempre ha sido fácil. El diseño de la propia AIE ha sido criticado por ser institucionalmente obsoleto, ya que no incluye a consumidores clave como China o la India (Miller, 2011; Van de Graaf, 2012). Además, la estructura de voto de su Comité de Reservas Estratégicas se basa principalmente en los niveles de importaciones de petróleo de los países miembros en 1973. Aunque en la década de 1990 hubo varios esfuerzos para redistribuir los votos todos ellos resultaron fallidos (Bamberger, 2004). Más recientemente, la decisión de liberar reservas estratégicas, supuestamente 
para compensar la pérdida de producción de Libia pero de hecho para presionar a la OPEP para que aumentase su producción, encontró la resistencia de algunos países europeos. Las reservas estratégicas fueron liberadas en el verano de 2011, pero varios miembros europeos de la AIE han venido resistiendo con éxito peticiones similares en los años siguientes por parte de los EEUU, interesados en vender parte de las ingentes reservas estratégicas acumuladas por su reciente bonanza petrolera no convencional.

La única institución formal donde los consumidores y productores de hidrocarburos se reúnen es el Foro Internacional de Energía (IEF), un acuerdo intergubernamental que agrupa a los signatarios de la Carta del IEF. Más que una organización internacional se trata de una reunión bianual de ministros de energía con una secretaría permanente con sede en Riad desde 2003, encargada de organizar las reuniones ministeriales y fomentar la transparencia de los datos de energía a través de la Joint Data Oil Initiative-JODI (Fattouh y van der Linde, 2011). Algunos autores han propuesto que el IEF podría proporcionar bienes públicos como infraestructuras energéticas alternativas o de repuesto, e incluso el mantenimiento de capacidades ociosas (Harks, 2010). En el ámbito cooperativo entre productores y consumidores, a los que suma los países de tránsito, también está la Carta de la Energía (Energy Charter), que pese a haber sido incapaz de gobernar las exportaciones de gas ruso ha recibido mucha atención académica (Konoplyanik y Wälde, 2006; Herranz y Natorski, 2012 ).

Fuera del campo de los hidrocarburos hay instituciones tradicionales dedicadas a hacer frente a los retos trasnacionales planteados por la energía nuclear, como la IAEA o la Agencia de Energía Nuclear de la OCDE (NEA) (Fischer, 1997; Baker y Stoker, 2013). Pero las cuestiones energéticas globales trascienden el diálogo y las tensiones entre los productores, consumidores y países de tránsito. Hay temas nuevos, como las energías renovables, la sostenibilidad, la pobreza energética, la eficiencia o el buen gobierno de los recursos. Estos nuevos asuntos de la agenda energética global han dado lugar a lo que se puede llamar proto-regímenes en áreas como la eficiencia energética (IPEEC), las energías renovables (IRENA) o la gobernanza de los recursos (EITI) (Lesage et al., 2010a; Colgan et al., 2013).

La Agencia Internacional de Energías Renovables (IRENA) fue fundada en 2009 después de varias conferencias y bajo los auspicios de los principales productores de energía renovable. Sostenibilidad y pobreza energética entraron en la agenda internacional a través de varias iniciativas, como la iniciativa de la ONU SE4ALL (Sovacool, 2012). El buen gobierno de los recursos energéticos ha sido objeto de considerable atención en los últimos años, dando lugar a iniciativas como la Iniciativa de Transparencia de las Industrias Extractivas (EITI), o la Carta de los Recursos Naturales (NRC) (Carbonnier, 2011). Además, un elemento clave de las políticas energéticas nacionales como los subsidios ener- 
géticos ha sido abordado por el G-20 (Kim y Chung, 2012; Lesage y Van de Graaf, 2013).

La creciente agenda de la gobernanza energética global reproduce la habitual pauta de la cooperación internacional de avanzar en paralelo en la profundización y ampliación, abarcando niveles más profundos de coordinación y extendiéndolos a nuevos temas. Los acuerdos internacionales relacionados con la energía adoptan modelos muy diferentes: organizaciones intergubernamentales como la AIE, la OPEP y la OPAEP, que en el caso de la OPEP actúa también como un cartel y en el de la OCDE incluye agencias dedicadas como la NEA o la propia AIE; estándares globales como la EITI o NRC ; plataformas y foros de diálogo, como el IEF que ha llevado a acuerdos diferenciados como la iniciativa JODI sobre la transparencia en las estadísticas energéticas, el IPEEC o el GECF; y las organizaciones de Naciones Unidas relacionadas con temas energéticos internacionales como el IAEA o iniciativas como E4ALL. A esto se suma la dimensión regional, con sistemas regionales de cooperación energética que continúan desarrollándose con mayor o menor éxito, principalmente en torno a la UE (Comunidad del Tratado de la Energía-ECT) pero también en América Latina (OLADE, Petrocaribe) .

Todos estos temas, viejos y nuevos, no son abordados por un único régimen internacional pese a las demandas en favor de la creación de una Agencia Global de la Energía (El-Baradei, 2008). Naciones Unidas ha tenido éxito en la creación de un régimen para la energía nuclear (IAEA), pero los gobiernos han sido reacios a abordar otras dimensiones internacionales de la energía, dejando un vacío normativo e institucional en materia de gobernanza energética global (Karlsson-Vinkhuyzen, 2010). Siguiendo a Ostrom (2010), algunos autores caracterizan la estructura energética mundial como un sistema policéntrico complejo y apelan a la necesidad de crear una estructura de gobernanza igualmente policéntrica con vínculos más fuertes entre los acuerdos ya existentes (Cherp et al., 2011). O incluso un Consejo de Estabilidad Energética que establezca las normas de las inversiones energéticas a nivel global, siguiendo el modelo del Consejo de Estabilidad Financiera y las normas de Basilea para el sector financiero (Victor y Yueh, 2010).

Algunas de las instituciones o regímenes mencionados, formales o informales, están vinculados a las capacidades materiales (como la OPEP, la AIE o el IEF). Otras en cambio reflejan factores ideacionales, caso de la EITI, NRC, IPEEC, E4ALL, o la iniciativa del G-20 sobre los subsidios. Otras incorporan tanto ideas como capacidades, como IRENA. Algunas de ellas se solapan, como EITI y NRC o IRENA y E4ALL, y la mayoría interactúan entre sí de manera poco estructurada. El resultado está lejos de constituir un régimen internacional energético homogéneo, tal y como lo concibe la economía política internacional, edificado sobre una balanza inequívoca de poder energético. Se trata más 
bien una nube de diferentes disposiciones y arreglos institucionales que se ocupan de los diversos aspectos de la gobernanza energética global. La energía se gobierna de manera fragmentada (piecemeal) y sobre todo mediante respuestas ad-hoc (Florini y Sovacool, 2009; Palazuelos, 2012) .

En este contexto, la hegemonía se distribuye entre países productores, consumidores y de tránsito y, dentro de ellos, de manera diferenciada según las fuentes de energía (petróleo, gas, nuclear y renovables). Por ejemplo, la OPEP ha ejercido la hegemonía en el suministro de petróleo y los miembros de la AIE en la demanda de petróleo y gas. Ambos cooperaron para crear el IEF, pero la efectividad del mismo ha quedado limitada a la promoción del diálogo y la confianza y no ha sido concebida como un régimen internacional para la energía (Fattouh y van der Linde, 2011). Por otra parte, la cuestión de identificar quién ostenta la hegemonía en el seno de cada arreglo institucional específico, como el dominio de la AIE por parte estadounidense o de la OPEP por Arabia Saudita, no es una tarea fácil en la medida en que a menudo dicha posición dominante puede ser discutida, en ocasiones con éxito. Las cuestiones relacionadas con la hegemonía también han surgido en la creación de instituciones como la IAEA, promovido por los EEUU tras la IIGM (Fischer, 1997); o la misma IRENA, impulsada por los principales productores de energías renovables, insatisfechos con lo que consideraban excesivo énfasis de la AIE en los combustibles fósiles (Colgan et al., 2013; Van de Graaf, 2013).

La hegemonía también está limitada por la interacción de las diferentes instituciones que integran los distintos niveles de gobernanza global de la energía. La influencia de la OPEP en los mercados del petróleo se ha visto reducida por la existencia de la AIE; la capacidad del IEF para lograr una cooperación eficaz se ha visto obstaculizada por la divergencia en las preferencias de productores y consumidores; el liderazgo de la AIE y la OCDE en materia de energías renovables fue contestada por la creación de IRENA; y la AIE no puede pretender controlar la seguridad de suministro del petróleo (ni del gas) ya que la demanda mundial de energía se ha desplazado hacia países emergentes como China o la India. Por último, la aparición de nuevos arreglos institucionales relacionados con la sostenibilidad y las energías renovables (Pires Manso y Bashiri Behmiri, 2013), la eficiencia, la gobernanza de los recursos naturales y la pobreza energética restringen el espacio de política de las instituciones establecidas en sus respectivas áreas.

En definitiva, la fragmentación de la gobernanza energética global hace que sea difícil evaluar si las ideas, las instituciones y las capacidades materiales están alineadas a nivel agregado, porque su equilibrio varía en las distintas áreas temáticas y arreglos institucionales respectivos. En este contexto, la hegemonía está restringida institucionalmente. La limitación principal es el funcionamiento de los propios mercados energéticos, que disciplina los objetivos de los acuer- 
dos mencionados. Por ejemplo, el GECF no ha encontrado la manera de influir en los mercados de gas debido a la propia estructura de los mismos: regionales en vez de globales, indexados al petróleo y gestionados por contratos a largo plazo (Wagbara, 2007). Y mientras que la OPEP y la AIE han logrado estabilizar el mercado del crudo en algunos momentos, no han sido capaces de contradecir los fundamentos de la oferta y la demanda de manera indefinida (Goldthau y Witte, 2011; Miller, 2011).

\section{EL COMPLEJO DE REGÍMENES ENERGÉTICOS}

Sin embargo, los mercados energéticos requieren instituciones para preservar las reglas del juego, reducir los costes de transacción y fijar estándares: tampoco en el campo de la energía global puede haber "mercados sin instituciones" que los sustenten (Goldthau y Witte, 2010:12). En ausencia de un marco institucional homogéneo, los problemas energéticos mundiales o regionales, complejos e interconectados, no pueden ser abordados de manera consistente por los mercados, los gobiernos, las organizaciones intergubernamentales y otros arreglos institucionales existentes (Florini y Sovacool, 2011). Por otra parte, la energía es un 'mega-problema' (mega-issue) profundamente arraigado en otros sectores y políticas con los que tiene vínculos importantes, como son los relacionados con las cuestiones económicas, ambientales y de seguridad (Lesage et al., 2010b).

En lo que respecta a la gobernanza, la energía es diferente a otros asuntos globales económicos como el comercio o las inversiones. Las diferencias se dan en varios aspectos: una gran complejidad horizontal y vertical, costes elevados y prevalencia de la dependencia de la senda, por lo que las externalidades globales relacionadas con la energía resultan más complejas de gestionar y los bienes públicos respectivos más difíciles de proveer (Goldthau y Sovacool, 2012). La fragmentación en diferentes marcos institucionales es la característica central de la gobernanza global de la energía (Cherp et al., 2011), lo que refleja la desigual distribución de capacidades materiales e ideacionales en los diferentes temas que conforman su agenda.

A ello se añade que la gobernanza energética es cada vez más compleja en un mundo crecientemente multipolar. Lesage et al. (2010b) han señalado que sólo los acuerdos internacionales energéticos que reflejen esa creciente multipolaridad pueden aportar un valor añadido a la gobernanza global, principalmente a través del liderazgo en la provisión de bienes públicos globales relacionados con la energía. Sin embargo, su propuesta consiste en que agrupaciones ya existentes como el G20 se centren en temas energéticos en lugar de crear un régimen internacional energético en sí. Otros autores han puesto de manifiesto que la falta de prioridades claras para los objetivos de la gobernanza energética global dificulta la coordinación (Dubash y Florini, 2011). Este es también el 
caso tanto con el G-8 y el G-20, que carecen de mecanismos para poner en práctica la acción colectiva y relacionarse con los países no miembros de una manera estructurada (Lesage et al., 2009; Van de Graaf y Westphal, 2011).

En realidad, la caracterización del panorama energético global como un mundo multipolar no hace justicia a la complejidad de la realidad internacional. Al menos en aquellas zonas en las que los mercados (mundiales o regionales) son la fuerza principal de regulación de los flujos energéticos, la tendencia se puede abordar con mayor precisión mediante el concepto de inter-polaridad (Grevi, 2009). El panorama energético global se caracteriza tanto por la multipolaridad como por un rápido aumento de la interdependencia de los mercados energéticos. Esta precisión no es (sólo) una sutileza académica en la medida en que conlleva implicaciones políticas claras: la gobernanza global de la energía debe abordarse como la gestión de la interdependencia en un contexto de acelerada redistribución relativa del equilibrio mundial de poder. Una vez más, esta reconfiguración geopolítica tiene implicaciones relevantes para la gobernanza energética global.

En resumen, la interacción de los diferentes componentes del complejo de regímenes existente hace que sea difícil establecer un cuadro claro de alineación hegemónica entre capacidades materiales, ideas e instituciones. Las divergencias ya no se dan sólo entre productores y consumidores, sino que las preocupaciones sobre pobreza energética, buena gobernanza de los recursos, el problema de la eficiencia o la sostenibilidad, actúan como factor limitativo a lo que puede lograr la cooperación consumidores-productores. La hegemonía se ve limitada por la interacción de actores, mercados y acuerdos internacionales, y la innovación institucional a veces requiere la agregación heterogénea tanto de capacidades materiales como de ideas.

Para estos casos Raustiala y Victor (2004: 279) introdujeron el concepto de 'complejo de regímenes' (regime complex), definido como aquel "conjunto de instituciones no jerárquicas que se superponen parcialmente y que rigen un área particular". El concepto se utilizó por primera vez para tratar la gobernanza mundial de los recursos fito-genéticos, pero después se ha aplicado a cuestiones más cercanas al objeto de este artículo, como el cambio climático (Abbott, 2012) o la propia gobernanza de la energía (Colgan et al., 2012). Orsini et al. (2013: 29) proponen una definición alternativa y más estructurada que encaja en el diagnóstico anterior:

"Una red de tres o más regímenes internacionales que se refieren a un tema común; con membresías superpuestas; y que generan interacciones sustantivas, normativas u operativas reconocidas como potencialmente problemáticas si no son gestionadas de manera eficaz".

Colgan et al. (2012 ) caracterizan el cambio institucional en el 'complejo energético de regimenes' como un proceso de 'equilibrio puntuado' (punctuated 
equilibrium) con períodos sin ninguna innovación significativa seguidos de otros de gran innovación, en vez de una transformación gradual en el tiempo (Krasner, 1984). Para ellos, la innovación institucional aparece cuando una parte suficientemente grande de la coalición de estados no está satisfecho con el resultado del conjunto actual de las instituciones que gobiernan cualquier cuestión energética particular. Pero también concluyen que los altos niveles de insatisfacción de los consumidores o productores, medida por los precios del petróleo ${ }^{2}$, son una condición necesaria pero no suficiente para la innovación institucional:

"El carácter y grado de cambio institucional en las políticas energéticas depende del grado de homogeneidad de las preferencias entre los actores clave en las instituciones existentes. Cuando se ha dado homogeneidad en los intereses de los principales actores, el resultado ha sido un cambio institucional dependiente de la senda. Cuando ha habido heterogeneidad en los intereses, los estados descontentos han tenido que crear nuevas instituciones sin predecesores obvios, porque las normas existentes permiten a sus oponentes bloquear los cambios importantes en las instituciones existentes" (Colgan et al., 2012: 139).

Sin embargo, la medición de la insatisfacción a través de los precios del petróleo limita el análisis a la dinámica entre consumidores y productores en los mercados, dejando de lado otras fuentes de energía y cuestiones ajenas al diálogo entre productores y consumidores, como la buena gobernanza de los recursos energéticos o la lucha contra la pobreza energética y por la sostenibilidad. Esto es consistente con el enfoque de Kehoane (1984), que se basa en el poder (capacidades materiales) como el principal vector de formación de los regímenes internacionales. Este punto de vista ignora el hecho de que la mayoría de los nuevos temas de la agenda global de la energía están relacionados con vectores ideacionales más que materiales. No obstante, hay que reconocer que la afirmación de Kehoane (2009) de que las iniciativas impulsadas por factores ideacionales permanecen hoy en la marginalidad es esencialmente correcto también para el 'complejo energético de regímenes'.

La razón es que sus instituciones centrales nacieron con la intención de proveer un bien público colectivo, seguridad energética: seguridad de suministro para los países consumidores y seguridad de demanda para los productores. Otros bienes públicos como la sostenibilidad, el desarrollo energético sostenible, el buen gobierno de los recursos energéticos y la eficiencia energética no se consideraron como objetivos hasta hace poco, y sólo de manera renuente. De hecho, para las compañías petroleras internacionales y nacionales, así como para las autoridades económicas, esas metas eran percibidas como conflictivas

\footnotetext{
${ }^{2}$ Los países importadores están insatisfechos cuando los precios suben abruptamente o se mantienen elevados; y los exportadores cuando los precios caen fuertemente o permanecen bajos.
} 
con el objetivo de la seguridad energética entendida de manera convencional. Aunque el progreso de estas metas en la agenda energética global ha sido modesto, su mera aparición constituye un cambio hacia un concepto más inclusivo y global de la seguridad energética.

Como ya se ha avanzado, los diferentes componentes del 'complejo energético de regímenes' pueden ser aproximados por los arreglos institucionales dedicados a proveer los siguientes bienes públicos colectivos:

- Seguridad del suministro de los países consumidores: OCDE, AIE y G-7.

- Seguridad de demanda, conforme a las preferencias de los países productores de la OPEP, GECF y OPAEP.

- Seguridad energética cooperativa, relacionada con el diálogo entre productores y consumidores en materia de transparencia estadística e infraestructuras de transporte: IEF, G-8 y G-20, así como redes informales del tipo Consejo Mundial de Energía (WEC) y el Consejo Mundial del Petróleo (WPC).

- Cooperación en energía nuclear: IAEA y NEA.

- Despliegue Energías renovables: IRENA, E4ALL, Rio +20.

- Eficiencia energética: IPEEC y E4ALL.

- Buen gobierno de los recursos energéticos: EITI, NRC y PWYP, pero también los nuevos estándares estadounidenses y comunitarios de trnasparencia en industrias extractivas.

- Desarrollo energético y erradicación de la pobreza energética: E4ALL.

Esta imagen dista de ser completa, y no sólo porque la lista de arreglos e iniciativas del anexo 1 no pretende ser exhaustiva. Las compañías energéticas, las ONG y las comunidades epistemológicas están inter-conectadas por redes formales e informales, y los gobiernos tienen sus propias iniciativas transnacionales en materia energética. Sin embargo, mediante la selección de los arreglos institucionales más relevantes ofrece un primer mapa de los diferentes componentes del 'complejo energético de regímenes'. Las agrupaciones regionales con contenido energético también son una parte importante del mismo, pero su tratamiento excede el objeto de estas páginas. Mientras que algunas de ellas, como OLADE y Petrocaribe en América Latina tienen una orientación básicamente introvertida, las iniciativas energéticas de la UE trascienden el ámbito de la política energética europea para tratar de influir no sólo en la gobernanza energética de su vecindad, sino también con la promoción de normas de vocación global (Escribano, 2011; Herranz y Natorski, 2012). 


\section{LA PROVISIÓN DE BIENES PÚBLICOS GLOBALES ENERGÉTICOS}

Los bienes públicos globales sólo han entrado recientemente en la literatura dedicada a la gobernanza de la energía, y de manera casi exclusiva en lo que respecta a la seguridad energética (Escribano y García-Verdugo, 2011; Goldthau, 2012). La consideración de la seguridad energética como bien público global es un tema tan discutido como la validez operativa del propio concepto. De hecho, el buen gobierno de los recursos energéticos, la lucha contra el cambio climático o el desarrollo energético sostenible fueron primero propuestos como bienes intermedios para garantizar la seguridad energética global a largo plazo, pero desde entonces han adquirido la entidad de bienes públicos globales en sí mismos. La enumeración de la sección precedente lleva a considerar la naturaleza del bien público global (o regional o colectivo) que un determinado arreglo institucional tiene como objetivo proporcionar y las tecnologías correspondientes para su provisión.

Este es un punto crítico para evaluar la hegemonía limitada que caracteriza la gobernanza energética global, puesto que la naturaleza del bien público determina si una estructura hegemónica es viable o no para su provisión, una intuición ya presente en Kindleberger (1986). Sandler (2003) analiza diferentes (siete) tecnologías de agregación para la provision de bienes públicos globales, en función de cómo las contribuciones individuales proveen la cantidad total del bien público, sea éste puro e impuro, bien de club o product conjunto (joint product). El mismo análisis puede llevarse a cabo para los bienes públicos regionales (Sandler, 2006). En este artículo se limita el análisis a cuatro tecnologías de agregación: suma (summation), eslabón más débil (weakest-link), umbral (threshold) y tecnologías de agregación del mejor disparo (best-shot).

El agregador más tradicional es la suma, en la que el nivel total de bien público es igual a la suma de las contribuciones de los agentes. Por ejemplo, desde la perspectiva del consumidor, la liberación de reservas estratégicas de los miembros de la AIE se añade de manera acumulativa (Goldthau , 2012). Desde la perspectiva del proveedor, el endurecimiento de las cuotas de la OPEP supone una contribución negativa en términos de producción, lo que implica una contribución positiva en términos de pérdida de ingresos. La suma supone que cada contribución es perfectamente sustituible por las de los demás, ofreciendo incentivos al free-riding: consumidores de petróleo no miembros de la AIE (incluso grandes consumidores como China o la India) se beneficiarían de los efectos en los precios de la liberación por parte de la AIE de sus reservas estratégicas sin contribuir a su mantenimiento; los productores no OPEP y los imcumplidores de ésta podrían beneficiarse igualmente de cualquier aumento de los precios del petróleo sin sacrificar su producción. En ambos casos, el com- 
promiso requiere un acuerdo internacional como la AIE o la OPEP para superar el free-riding.

Un agregador 'del más débil'-weakest link se aplica cuando la contribución más pequeña fija el nivel global de la provisión del bien público. El ejemplo típico es la integridad de un corredor energético dado, como un gasoducto, una red eléctrica o un estrecho marítimo, que dependen de la integridad de su punto más débil. El bloqueo o ralentización de los corredores marítimos abiertos, como los que unen el Golfo Pérsico con Europa y los EEUU a través del Canal de Suez o el estrecho de Ormuz, o de un gasoducto del norte de África por conflictos en países productores como Libia o Egipto, o países de tránsito como Túnez o Marruecos, suponen eslabones débiles a lo largo de toda la cadena de suministro.

El agregador weakest-link puede aplicarse también a la producción y el consumo de petróleo: Iraq es un productor siempre promisorio incapaz de materializar en producción sus enormes reservas debido a la inestabilidad política y la violencia. En el mismo sentido, la ausencia de suficientes reservas estratégicas en consumidores clave, como China o la India, podría generar o empeorar una crisis de precios en los mercados mundiales del petróleo. Los recientes acontecimientos de Ucrania muestran las externalidades negativas potenciales para la UE del débil eslabón que este país supone en los corredores de gas procedentes de Rusia.

El agregador de umbral se aplica cuando los beneficios de la provisión del bien público sólo aparecen una vez que la cantidad acumulada del mismo alcanza un cierto nivel. En los ejemplos anteriores, cuando los barriles liberados de reservas estratégicas o retirados mediante la reducción de cuotas de la OPEP son suficientes para estabilizar el mercado petrolero en el nivel mínimo deseado. Si se aplica un agregador de umbral, por lo general es necesaria capacidad de liderazgo para aunar esfuerzos y no ofrece incentivos al free-riding; sin embargo, una vez que el umbral se alcanza el incentivo al free-riding plantea los mismos problemas que el agregador de suma.

Por último, los agregadores 'best shot' son aquellos en los que la provisión del bien está determinada únicamente por la mayor contribución. Por ejemplo, el 'mejor tirador' para estabilizar el mercado del petróleo es Arabia Saudita. Se trata del único productor con la capacidad ociosa requerida para aumentar significativamente la producción en el corto plazo, y el único que puede permitirse la reducción de la producción e incurrir en pérdidas de ingresos. Y ello pese a que desde la primavera árabe los precios del petróleo que equilibran su balanza fiscal y externa han aumentado constantemente. Con tecnologías de agregación tipo best shot y weakest link, Harrison y Hirschleifer (1989) han demostrado que los agentes con una fuerte preferencia por igualar las contribuciones de los demás tienden a transformar el dilema social planteado en un juego de coordi- 
nación. Esto ofrece un enfoque más cooperativo de la seguridad energética y de la provisión de otros bienes públicos globales relacionados con la energía.

Todos los ejemplos anteriores están deliberadamente relacionados con las interacciones entre productores y consumidores, incluyendo la mediación de los países de tránsito, e implican capacidades materiales bien para estabilizar el mercado de petróleo o para asegurar las infraestructuras de producción y transporte. Esas capacidades pueden ser contingentes, como liberar (o no) reservas o emplear (o no) capacidad ociosa, pero suponen el poder real (la capacidad y la voluntad de asumir un costo dado) para influir eficazmente en el mercado del petróleo. Sin embargo, los complejos de regímenes tienen también un contenido normativo (Orsini, 2013), y la innovación institucional puede ser impulsada por nuevas ideas sin gran base material, como sostiene Cox (1996). Este es precisamente el caso de arreglos como EITI, E4ALL, IPEEC y en cierta medida IRENA, todos ellos de contenido básicamente ideacional y muy relacionado con la provisión de los bienes públicos respectivos. Sin embargo, el enfoque público global en materia energética prácticamente se ha mantenido limitado a los asuntos relacionados con la energía sostenible (Karlsson-Vinkhuyzen et al., 2012).

Tales arreglos institucionales, ya sean iniciativas, normas, foros u organizaciones internacionales, se ocupan de diferentes bienes públicos que se correlacionan con tecnologías de agregación distintas. La iniciativa EITI está cerca de una tipología cercana al weakest-link, porque la mejora de la gobernanza de los recursos energéticos en los productores peor gobernados, y eso puede ser una de las vías óptimas para mejorar el nivel global de gobernanza de dichos recursos. Lo mismo se aplica a la iniciativa E4ALL, en la medida en que la promoción del desarrollo energético sostenible a nivel mundial enfoca la lucha contra la pobreza energética en los países más afectados, mejorando así de la manera más eficaz la pobreza energética global. Por el contrario, la provisión de IRENA procede principalmente por la suma de las contribuciones financieras (o las capacidades de generación renovable) de sus miembros.

Por otro lado, la legislación de EEUU y de la UE en la mejora de la transparencia y la divulgación de los pagos de las compañías energéticas a los gobiernos productores es una estrategia del tipo best-shot, en la medida en que sea capaz de establecer un estándar global para la transparencia en las industrias extractivas respetado por terceros países. La eficiencia es también más cercana a una tecnología del tipo best-shot, porque hay países altamente ineficientes en el consumo de energía, como los EEUU o los grandes productores de hidrocarburos, que podrían reducir fácilmente las prácticas ineficientes. Un caso sometido a compromisos internacionales es la quema del gas o flaring, otro candidato a tecnologías selectivas del tipo best-shot. Todo esto implica que los distintos bienes públicos globales relacionados con la energía pueden requerir tecno- 
logías de provisión alternativas o incluso complementarias (como EITI y los estándares de transparencia estadounidenses y comunitarios), y por tanto arreglos institucionales diferenciados conforme a la variedad de incentivos existentes para la cooperación.

Si se consideran conjuntamente los enfoques de Cox (1996) y Kehoane (1984), las instituciones son de alguna manera la proyección combinada de capacidades materiales e ideacionales. Desde una perspectiva de bienes públicos globales (o regionales), constituyen bienes públicos intermedios cuya provisión requiere preferencias homogéneas entre varios proveedores, dependiendo de la tecnología de agregación asociada a cada bien público final. De hecho, buena parte del impulso ideacional viene por el lado de la demanda de nuevos arreglos institucionales dedicados a la provisión del bien público global final. En algunos casos, la hegemonía puede no ser suficiente (como la suma y los agregadores de umbral), mientras que en otros en otros podría ser irrelevante (situaciones weakest-link). Pero los 'mejores tiradores' (o agentes más capaces, como suele traducirse en español) suelen ser hegemones energéticos (mundiales o regionales), y los agregadores de suma, umbral y weakest-link requieren algún tipo de liderazgo.

\section{CONSIDERACIONES FINALES}

La fragmentación de la gobernanza energética global caracteriza a ésta como un complejo de regímenes que abarcan aspectos diferenciados. La irrupción de nuevos temas ha ido expandiendo la agenda energética global, y el tránsito hacia una estructura inter-polar dificulta la alineación de preferencias cada vez más heterogéneas entre los principales actores energéticos mundiales. Las divergencias no se limitan a las existentes entre productores y consumidores, sino que se amplían a vectores ideacionales en los cuales las capacidades materiales no bastan para fijar la agenda. Por ello, las soluciones hegemónicas son difíciles de materializar y, en cualquier caso, representan hegemonías limitadas a determinados aspectos de la gobernanza energética global.

En esta estructura policéntrica de gobernanza el reforzamiento de los vínculos entre instituciones, formales o informales, resulta clave como sustituto de un único régimen internacional homogéneo. Cada una de esas instituciones o arreglos tiene por objeto suministrar un bien público global determinado, sea éste la seguridad de suministro (AIE), la de demanda (OPEP) o cualquiera de los nuevos bienes públicos de carácter normativo como la mejora de la eficiencia, la buena gobernanza de los recursos o la sostenibilidad, entre otros. La caracterización de los arreglos institucionales correspondientes como bienes públicos globales (o regionales) intermedios permite (i) elaborar un mapa en que cada institución constituye un instrumento (u objetivo intermedio) que se relaciona con un objetivo (el bien público final), y (ii) analizar en qué medida dichos 
arreglos son consistentes con la tecnología de provisión óptima para cada uno de esos bienes públicos.

La inclusión de este análisis por el lado de la oferta de los bienes públicos energéticos globales permite matizar los enfoques geopolíticos, de corte esencialmente neorrealista en tanto priman la competencia, con una perspectiva de gobernanza policéntrica más cercana a la narrativa neoliberal y por tanto más proclive a la cooperación.

\section{REFERENCIAS BIBLIOGRÁFICAS}

ABBOTT, K. W. (2012). "The transnational regime complex for climate change". Environment and Planning C: Government and Policy 30 (4): 571-590.

BAKER, K. and G. STOKER (2013). "Governance and Nuclear Power: Why Governing is Easier Said than Done". Political Studies, 61 (3): 580-598.

BAMBERGER, C. S. (2004). History of the IEA: The First 30 Years. Paris: IEA.

BARBÉ, E. and O. COSTA (2014). "The EU in an unsettled international system. Coping with the power shift in global governance". Paper presented to the Barcelona Workshop on Global Governance organized by ESADEgeo and IBEI (Barcelona, January 9-10). http://www.eu-ianus.eu/

CARBONNIER, G. (2011). "The Global and Local Governance of Extractive Resources". Global Governance, 17 (2): 135-147.

CHERP, A., J. JEWELL and A. GOLDTHAU (2011) "Governing Global Energy: Systems, Transitions, Complexity". Global Policy (2) 1: 75-88.

COLGAN, J. D., R. O. KEOHANE and T. VAN DE GRAAF (2013). "Punctuated equilibrium in the energy regime complex", Review of International Organizations, 7 (2): 117-143.

CORRELJÉ, A. y C. VAN DER LINDE (2006). "Energy Supply Security and Geopolitics: A European Perspective". Energy Policy 34 (5): 532-543.

COX, ROBERT W. (1996). Approaches to World Order [with Timothy J. Sinclair], Cambridge: Cambridge University Press.

DUBASH, N. K. and A. FLORINI (2011). "Mapping Global Energy Governance". Global Policy, Special Issue on Global Energy Governance, 2 (S1): 6-18.

EL-BARADEI, M. 2008. A global agency is needed for the energy crisis, Financial Times July 23, acceded on-line December 27, 2013: http://www.ft.com/cms/s/0/b3630dd058b5-11dd-a093-000077b07658.html\#axzz1Fw0wO0rv.

ESCRIBANO, G. (2010). "Convergence towards Differentiation: The Case of Mediterranean Energy Corridors”. Mediterranean Politics, 15 (2): 211-230.

ESCRIBANO, G. (2011). "Markets or Geo-politics? The Europeanization of Euro-Mediterranean Energy Corridors". Journal of Energy Sector Management", 5 (1): 39-59.

ESCRIBANO, G. and J. GARCÍA-VERDUGO (2011). "Energy security, energy corridors and the geopolitical context: a conceptual approach". In Marín-Quemada et al., eds. (2011): 26-37.

FATTOUH, B. and C. VAN DER LINDE (2011). The International Energy Forum: Twenty 
years of producer-consumer dialogue in a changing world. Riyadh: IEF.

FIORENTINI, R. and G. MONTANI (2012). The New Global Political Economy. From Crisis to Supranational Integration. Northampton, MA: Edward Elgar.

FISCHER, D. (1997). History of the International Atomic Energy Agency : the first forty years. Vienna : International Atomic Energy Agency.

FLORINI, A. and N. K. DUBASH (2011). "Introduction to the Special Issue: Governing Energy in a Fragmented World". Global Policy, 2, s1: pp. 1-5.

FLORINI, A. and SOVACOOL, B.K. (2009). "Who governs energy? The challenges facing global energy governance". Energy Policy 37: 5239-5248.

FLORINI, A., and B. K. SOVACOOL (2011). "Bridging the Gaps in Global Energy Governance". Global Governance: A Review of Multilateralism and International Organizations, 17 (1): 57-74.

GIL-ALANA, L. A. and BARROS, C. P. (2011). "An analysis of oil production by OPEC countries: persistence, breaks, and outliers". Energy Policy, 39 (1): 442-453.

GOldTHAU, A. (2012). "A Public Policy Perspective on Global Energy Security". International Studies Perspectives, 13 (1): 65-84.

GOLDTHAU, A. and B. K. SOVACOOL (2012). "The uniqueness of the energy security, justice, and governance problem". Energy Policy 41: 232-240.

GOLDTHAU, A., and J. M. WITTE (2009). "Back to the future or forward to the past? Strengthening markets and rules for effective global energy governance". International Affairs 85 (2): 373-390.

GOLDTHAU, A., and J. M. WITTE, eds. (2010). Global Energy Governance. The New Rules of the Game. Berlin: Global Public Policy Institute and Brookings Institution Press.

GOldTHAU, A., and J. M. WITTE (2011). "Assessing OPEC's Performance in Global Energy". Global Policy, Special Issue on Global Energy Governance, 2, Issue Supplement s1: 31-39.

GREVI, G. (2009). "The interpolar world: a new scenario". Occasional Paper 79, European Union Institute of Security Studies.

HARRISON, G. W. and J. HIRSCHLEIFER (1989). "Experimental Evaluation of Weakest Link/Best Shot Models of Public Goods”. Journal of Political Economy, 97: 201-223.

HARKS, E. (2010). "The IEF and the Mitigation of Oil Market Risks". In Goldthau and Witte (eds.). Brookings Institution Press: Washington DC.

HERRANZ, A. and M. NATORSKI (2012). "The European energy policy towards Eastern neighbours: rebalancing priorities or changing paradigms". In: Morata, F. and I. Solorio (Eds) European Energy Policy: The environmental dimension. Cheltenham: Edward Elgar, pp. 179-203.

JOYCE, J. P. and T. SANDLER (2008). "IMF retrospective and prospective: A public goods viewpoint". Review International Organizations, 3: 221-238.

KARLSSON-VINKHUYZEN, S.I. (2010). "The United Nations and global energy governance: past challenges, future choices". Global Change, Peace and Security 22: 175-195.

KARLSSON-VINKHUYZEN S.I., N. JOLLANDS, and L. STAUDT (2012). "Global governance for sustainable energy: The contribution of a global public goods approach". Ecological Economics 83: 11-18. 
KAUL, I., I. GRUNBERG and M. A. STERN, eds. (1999). Global Public Goods. International Cooperation in the $21^{\text {st }}$ Century. Oxford University Press: New York.

KEHOANE, R. O. (1984). Cooperation and Discord in the World Political Economy. Princeton Univ. Press.

KEOHANE, R. O. (2009). "The old International Political Economy and the new". Review of International Political Economy, 16 (1): 34-46.

$\mathrm{KIM}$, J. A. and S-Y. CHUNG (2012). "The role of the G20 in governing the climate change regime". International Environmental Agreements: Politics, Law and Economics, 12 (4): 361-374.

KINDLEBERGER, Ch. P. (1986). "International Public Goods without International Government". American Economic Review, 76 (1): 1-13.

KONOPLYANIK, A. and T. WÄLDE (2006). "Energy charter treaty and its role in international energy". Journal of Energy and Natural Resources Law, 24 (4): 523-558.

KRASNER, S. D., ed. (1983). International Regimes. Cornell University Press, Ithaca, New York.

KRASNER, S. D. (1984). "Approaches to the State: Alternative Conceptions and Historical Dynamics". Comparative Politics, 16 (2): 223-246.

LESAGE, D., T. VAN DE GRAAF y K. WESTPHAL (2009). "The G8's role in global energy governance since the 2005 Gleneagles summit”. Global Governance, 15(2): 259-277.

LESAGE, D., T. VAN DE GRAAF y K. WESTPHAL (2010a). "G8+5 collaboration on energy efficiency and IPEEC: Shortcut to a sustainable future?". Energy Policy, 38 (11): 6419-6427.

LESAGE, D., T. VAN DE GRAAF y K. WESTPHAL (2010b). Global Energy Governance in a Multipolar World. Burlington: Ashgate.

LESAGE, D. y T. VAN DE GRAAF (2013). “Thriving in Complexity? The OECD System's Role in Energy and Taxation". Global Governance, 19(1): 83-92.

MANN, Y. (2012). "Saudi Arabia's policy toward non-OPEC countries". Diplomacy and Statecraft, 23 (2): 381-391.

MAÑÉ, A. (2006). "European Energy Security: Towards the Creation of the Geo-energy Space". Energy Policy, 34: 3773-3786.

MARÍN-QUEMADA, J.M., J. GARCÍA-VERDUGO, and G. ESCRIBANO, eds. (2011). Energy Security for the EU in the 21st Century. Markets, Geopolitics and Corridors. New York/Oxon: Routledge.

MILLER, R. G. (2011). "Future oil supply: The changing stance of the International Energy Agency". Energy Policy, 39 (3): 1569-1574.

OSTROM, E. (2010). "Beyond Markets and States: Polycentric Governance of Complex Economic Systems". The American Economic Review, 100 (3): 641-672.

ORSINI, A., J. F. MORIN and O. YOUNG (2013). "Regime Complexes: A Buzz, a Boom, or a Boost for Global Governance?". Global Governance, 19 (1): 27-39.

OYE, K. A. (1986). "Cooperation under Anarchy”. Princeton University Press: Princeton.

PALAZUELOS, E. (2012). "Current oil (dis)order: players, scenarios, and mechanisms". Review of International Studies, 38: 301-319.

PIRES MANSO, J. R. y N. BASHIRI BEHMIRI (2013). "Renewable Energy and Sustainable Development", Estudios de Economía Aplicada, 31: 7-34. 
RATHBUN, B. C. (2011). "Before Hegemony: Generalized Trust and the Creation and Design of International Security Organizations". International Organization, 65, 2: 243-273.

RAUSTIALA, K. and VICTOR, D. (2004). "The Regime Complex for Plant Genetic Resources". International Organization, 58 (2): 277-309.

SANDLER, T. (2003). "Assessing the optimal provision of public goods: In search of the Holy Grail". In I. Kaul, P. Conceicao, K. Le Goulven and R.U. Mendoza (Eds.) Providing Global Public Goods: Managing globalization. New York: Oxford University Press, 131-151.

SANDLER, T. (2006). "Regional public goods and international organizations". Review of International Organizations 1 (1): 5-25.

SOVACOOL, B.K. (2012). "The political economy of energy poverty: A review of key challenges". Energy For Sustainable Development, 16 (3): 272-282.

VAN DE GRAAF, T. y K. WESTPHAL (2011). "The G8 and G20 as global steering committees for energy: opportunities and constraints". Global Policy, 2(S1): 19-30.

VAN DE GRAAF, T. (2012). "Obsolete or resurgent? The International Energy Agency in a changing global landscape". Energy Policy 48: 233-241.

VAN DE GRAAF, T. (2013). "Fragmentation in Global Energy Governance: Explaining the Creation of IRENA". Global Environmental Politics, 13 (3): 14-33.

VICTOR, D. G. y L. YUEH (2010). "The New Energy Order. Managing Insecurities in the Twenty-first Century". Foreign Affairs, 89 (1): 61-73.

WAGBARA, O. N. (2007). "How would the gas exporting countries forum influence gas trade?". Energy Policy, 35 (2): 1224-1237.

WIRL, F. (2012). "OPEC as a political and economical entity". European Journal of Political Economy, 25 (4): 399-408. 


\section{ANEXO}

\section{Principales arreglos institucionales energéticos}

\begin{tabular}{|c|c|c|}
\hline EITI & $\begin{array}{l}\text { Extractive Industries Transpa- } \\
\text { rency Initiative }\end{array}$ & $\begin{array}{l}\text { Estándar global lanzado por Blair en } 2002 \text { para promover la transpa- } \\
\text { rencia de los ingresos y la rendición de cuentas en el sector extrac- } \\
\text { tivo. Desarrollado y supervisado por una coalición de gobiernos, } \\
\text { empresas, sociedad civil, inversores y organizaciones internaciona- } \\
\text { les representadas en el Consejo de la EITI, apoyado por un Secreta- } \\
\text { riado Internacional con sede en Oslo. }\end{array}$ \\
\hline GECF & $\begin{array}{l}\text { Gas Exporting Countries } \\
\text { Forum }\end{array}$ & $\begin{array}{l}\text { Organización intergubernamental de } 11 \text { de los principales producto- } \\
\text { res de gas natural del mundo (Argelia, Bolivia, Egipto, Guinea Ecua- } \\
\text { torial, Irán, Libia, Nigeria, Qatar, Rusia, Trinidad y Tobago, y } \\
\text { Venezuela), lo que representa más del } 70 \% \text { de las reservas de gas } \\
\text { natural del mundo, } 38 \% \text { del comercio por tubería y el } 85 \% \text { del gas } \\
\text { natural licuado (GNL). Rusia, Irán y Qatar poseen en conjunto más } \\
\text { del } 55 \% \text { de las reservas mundiales de gas. Se estableció en Teherán } \\
\text { en } 2001 \text {, y la Oficina Ejecutiva y la Secretaría se basan en Doha, } \\
\text { Qatar. }\end{array}$ \\
\hline IAEA & $\begin{array}{l}\text { International Atomic Energy } \\
\text { Agency }\end{array}$ & $\begin{array}{l}\text { La OIEA, en sus siglas en español, es una organización internacional } \\
\text { independiente en relación al sistema de las Naciones Unidas, creada } \\
\text { en } 1957 \text { como centro de cooperación en el campo nuclear. Su } \\
\text { creación se remonta a la iniciativa Átomos para la Paz de Eisenho- } \\
\text { wer presentada a la Asamblea General de la ONU en 1953. Trabaja } \\
\text { con sus Estados miembros y socios en todo el mundo para promover } \\
\text { tecnologías nucleares seguras y pacíficas. La Secretaría tiene su } \\
\text { sede en Viena. }\end{array}$ \\
\hline AIE & $\begin{array}{c}\text { Agencia Internacional de la } \\
\text { Energía }\end{array}$ & $\begin{array}{l}\text { Organización intergubernamental con sede en París creada en el } \\
\text { marco de la OCDE en } 1974 \text {. Se dedicó inicialmente a responder a } \\
\text { las interrupciones del suministro de petróleo, así como a ser una } \\
\text { fuente de información sobre los mercados energéticos. Actualmente, } \\
\text { ha ampliado su enfoque a la seguridad energética, el desarrollo } \\
\text { económico y la protección medioambiental. Asesora las políticas } \\
\text { energéticas de los Estados miembros y coopera con terceros países } \\
\text { como China, India y Rusia. Sus miembros deben mantener reservas } \\
\text { estratégicas de petróleo equivalentes a } 90 \text { días de importaciones } \\
\text { netas del año anterior, y estar dispuestos a liberarlas en caso de } \\
\text { emergencia. La AIE ha liberado reservas estratégicas de petróleo en } \\
\text { tres ocasiones: en } 1991 \text { durante la Guerra del Golfo, en } 2005 \text { des- } \\
\text { pués del huracán Katrina, y en } 2011 \text { para compensar las interrupcio- } \\
\text { nes del suministro de petróleo de Libia. }\end{array}$ \\
\hline IEF & International Energy Forum & $\begin{array}{l}\text { Veinte años después de su primera reunión en París en julio de } \\
1991 \text {, el IEF ha evolucionado hasta convertirse en una de las plata- } \\
\text { formas más incluyentes para el diálogo productores-consumidores. } \\
\text { Ambos se reúnen regularmente para discutir asuntos de interés } \\
\text { común relacionados con la escena energética global. Es la reunion } \\
\text { periódica internacional más importante para los ministros de energía, } \\
\text { y sus participantes no sólo son miembros de la AIE y la OPEP, sino } \\
\text { que también asisten los principales actores energéticos globales, } \\
\text { como Brasil, China, India, México, Rusia o Sudáfrica. Los países } \\
\text { presents en el IEF representan más del } 90 \% \text { del suministro de } \\
\text { petróleo y gas y de su demanda global. Cuenta con una secretaría } \\
\text { permanente con sede en Riad, Arabia Saudita. }\end{array}$ \\
\hline IPEEC & $\begin{array}{l}\text { International Partnership for } \\
\text { Energy Efficiency Coopera- } \\
\text { tion }\end{array}$ & $\begin{array}{l}\text { Foro internacional fundado en } 2009 \text { para la cooperación en materia } \\
\text { de eficiencia energética. La cumbre del G-8 de junio } 2007 \text { aprobó } \\
\text { una propuesta de la UE para una iniciativa internacional sobre } \\
\text { eficiencia energética en colaboración con la AIE. Incluye a los países } \\
\text { del G8 más China, Corea del Sur, Brasil, México, India y la UE. La } \\
\text { Secretaría tiene su sede en la sede de la AIE en París. }\end{array}$ \\
\hline
\end{tabular}




\section{Principales arreglos institucionales energéticos (continuación)}

\begin{tabular}{|c|c|c|}
\hline JODI & $\begin{array}{l}\text { Joint Organisations Data } \\
\text { Initiative }\end{array}$ & $\begin{array}{l}\text { Un resultado del IEF, es una iniciativa destinada a mejorar la trans- } \\
\text { parencia de los mercados de materias primas energéticas globales } \\
\text { en interés de productores y consumidores. Se basa en el esfuerzo } \\
\text { conjunto de ambos y de las siete organizaciones asociadas (APEC, } \\
\text { Eurostat, AIE, OLADE, OPEP y UNSD) para proporcionar datos } \\
\text { energéticos y ayudar a mitigar las incertidumbres del mercado y la } \\
\text { volatilidad excesiva de los precios. }\end{array}$ \\
\hline NEA & Nuclear Energy Agency & $\begin{array}{l}\text { La OECE (predecesora de la OCDE) creó una Agencia Europea de } \\
\text { la Energía Nuclear (AEEN en español) en 1958. Su nombre fue } \\
\text { cambiado en } 1972 \text { para reflejar el creciente número de miembros no } \\
\text { europeos. Su primera tarea fue sentar las bases para la cooperación } \\
\text { nuclear y proporcionar un foro para la coordinación de los programas } \\
\text { nucleares nacionales de los países OCDE. En la década de 1970, } \\
\text { los gobiernos se vieron presionados a dar mayor prioridad a los } \\
\text { aspectos ambientales, de seguridad y de regulación. El colapso de la } \\
\text { URSS significó el acceso de nuevos países con reactores de diseño } \\
\text { soviético y una intense cooperación en mejorar sus estándares. }\end{array}$ \\
\hline OAPEC & $\begin{array}{l}\text { Organization of Arab } \\
\text { Petroleum Exporting } \\
\quad \text { Countries }\end{array}$ & $\begin{array}{l}\text { Organización multi-gubernamental con sede en Kuwait que pretende } \\
\text { coordinar las políticas energéticas en los países árabes productores } \\
\text { de petróleo. Diez días después de la Guerra de Yom Kippur de 1973, } \\
\text { Kuwait organizó reuniones en las que participaron tanto la OPAEP } \\
\text { como los miembros de la OPEP del Golfo Pérsico, entre ellos Irán. } \\
\text { La OPAEP redujo la producción de petróleo un } 5 \% \text { mensual durante } \\
\text { cinco meses induciendo la crisis del petróleo de } 1973 \text {. El embargo } \\
\text { fue la primera vez que los países productores de petróleo ejercieron } \\
\text { su capacidad energética con fines politicos de manera coordinada. } \\
\text { Sin embargo, un embargo similar liderado por OPAEP nunca ha } \\
\text { vuelto a ser posible por las divergencias en las preferencias políticas } \\
\text { y económicas de sus miembros. }\end{array}$ \\
\hline OPEP & $\begin{array}{l}\text { Organización de Países } \\
\text { Exportadores de Petróleo } \\
\text { (Organization of Petroleum } \\
\text { Exporting Countries) }\end{array}$ & $\begin{array}{l}\text { El cartel petrolero es bien conocido. Tiene su sede en Viena, y junto } \\
\text { con OPAEP desencadenó la crisis del petróleo de } 1973 \text {. Agrupa el } \\
73 \% \text { de las reservas probadas de petróleo del mundo y el } 43 \% \text { de la } \\
\text { producción petrolera. Arabia Saudita lidera el cartel (y el mercado } \\
\text { mundial) con el } 16 \% \text { de las reservas probadas de petróleo, el } 13 \% \\
\text { de la producción petrolera y una capacidad ociosa de alrededor de } 3 \\
\text { millones de barriles al día. }\end{array}$ \\
\hline SE4ALL & Sustainable Energy For All & $\begin{array}{l}\text { Iniciativa global liderada por el Secretario General de la ONU, Ban } \\
\text { Ki-moon, para lograr el acceso universal a la energía, mejorar la } \\
\text { eficiencia energética y aumentar el uso de energías renovables. } \\
\text { Lanzada en el año } 2011 \text {. }\end{array}$ \\
\hline
\end{tabular}

Fuente: Elaboración propia. 
